\title{
The Capacity of Some Pólya String Models
}

\author{
Ohad Elishco, Student Member, IEEE, Farzad Farnoud (Hassanzadeh), Member, IEEE, \\ Moshe Schwartz, Senior Member, IEEE, Jehoshua Bruck, Fellow, IEEE
}

\begin{abstract}
We study random string-duplication systems, which we call Pólya string models. These are motivated by DNA storage in living organisms, and certain random mutation processes that affect their genome. Unlike previous works that study the combinatorial capacity of string-duplication systems, or various string statistics, this work provides exact capacity or bounds on it, for several probabilistic models. In particular, we study the capacity of noisy string-duplication systems, including the tandem-duplication, end-duplication, and interspersedduplication systems. Interesting connections are drawn between some systems and the signature of random permutations, as well as to the beta distribution common in population genetics.
\end{abstract}

Index Terms-DNA storage, string-duplication systems, capacity, Pólya string models

\section{INTRODUCTION}

$\mathbf{S}$ EVERAL mutation processes are known, which affect the genetic information stored in the DNA. Among these are transposon-driven repeats [12] and tandem repeats which are believed to be caused by slipped-strand mispairings [18]. In essence, these mutation processes take a substring of the DNA and insert a copy of it somewhere else (in the former case), or next to the original copy (in the latter). In human DNA, it is known that its majority consists of repeated sequences [12]. Moreover, certain repeats cause important phenomena such as chromosome fragility, expansion diseases, gene silencing [20], and rapid morphological variation [8].

A formal mathematical model for studying these kinds of mutation processes is the notion of string-duplication systems. In such systems, a seed string (or strings) evolves over time by successive applications of mutating functions. For example, functions taking a substring of a string and copying it next to itself model mutation by tandem duplication. These stringduplication systems were studied in the context of formal languages (e.g., [14]) in an effort to place the resulting sets of mutated sequences within Chomsky's hierarchy of formal languages, as well as to derive closure properties.

In the context of coding theory, string-duplication systems were studied, motivated by applications to DNA storage in

The material in this paper was presented in part at the 2016 IEEE International Symposium on Information Theory.

Ohad Elishco is with the Department of Electrical and Computer Engineering, Ben-Gurion University of the Negev, Beer Sheva 8410501, Israel (e-mail: ohadeli@post.bgu.ac.il).

Farzad Farnoud (Hassanzadeh) is with the Department of Electrical and Computer Engineering, University of Virginia, Charlottesville, VA, 22903, USA, (email: farzad@virginia.edu). He was with the Electrical Engineering Department, California Institute of Technology.

Moshe Schwartz is with the Department of Electrical and Computer Engineering, Ben-Gurion University of the Negev, Beer Sheva 8410501, Israel (e-mail: schwartz@ee.bgu.ac.il).

Jehoshua Bruck is with the Department of Electrical Engineering, California Institute of Technology, Pasadena, CA 91125, USA (e-mail: bruck@paradise.caltech.edu). living organisms. In such a storage scheme, information is stored in the DNA of some organisms, and later read from them or their descendants [19]. This information, however, is corrupted by mutations. These include substitution errors, as well as insertions and deletions - all of which have already been extensively studied in the coding-theoretic community. However, another type of error is that of duplication, modeled mathematically by string-duplication systems.

Various aspects of string-duplication systems were studied, geared towards a comprehensive coding solution to duplication mutations. In [6], [9], the duplication mutation processes were treated as a source, and their exponential growth rate, i.e., their capacity, studied. This provided insights into the structure of error balls in the string-duplication channel. Some errorcorrecting codes for tandem duplication were presented in [10], [13]. The confusability of strings under tandem duplication was studied in [4], the mutation distance was bounded in [1], and more recently, [21] developed reconstruction schemes for uniform tandem duplication.

A drawback of all the papers mentioned above is a combinatorial (adversarial) approach, whereas we suspect a scenario involving DNA storage in living organisms must be probabilistic. To address this gap, a probabilistic model was studied in [5]. This model is not concerned with which mutated strings are possible, but rather with which are probable. With appropriate distributions applied to the choice of the mutated point, the mutation length, and its final position, we obtain an induced distribution on resulting strings. However, [5] was not able to provide any exact capacity calculation nor bounds, and managed to study only peripheral properties of the resulting string distributions, namely the frequencies of symbols and substrings.

Thus, the goal of this paper is to find the exact capacity of probabilistic string-duplication systems, or bound it. We also generalize the process to include noisy duplication. As we later see, even for very modest parameters this problem is extremely challenging.

The main contributions of this paper are an exact expressions for end-duplication systems and interspersed-duplication systems, for all noise parameters. Additionally, we find the exact capacity of noiseless tandem duplication and complement tandem duplication, and bound the capacity of the general noisy tandem-duplication system. In all cases we study duplication of length 1 only.

An important tool, widely used in the study of genetic drift in population genetics, is a Pólya urn model. It consists of an urn with balls of two different colors. In each step a ball is randomly (independently and uniformly) chosen and returned to the urn along with $k$ new balls of the same color [17]. There are many extensions to this model, where after each draw, a 
set of balls, whose number and composition depends on the color of the drawn ball, are put into the urn. However, in these models there is no structure on the balls in the urn and only the number of balls of each color matters. Thus, these models fail to apply to strings.

We therefore suggest extensions of the Pólya urn models to what we call Pólya string models, in which the balls form a string, which may be circular or linear, similar to bases of a DNA molecule. A step in this model typically involves choosing a random position (or equivalently a ball) in the string, where a modification to the string - the mutation occurs. In this paper, we focus on models in which after the draw, a sequence of balls is inserted to the string whose composition and position depend on the local properties of the string around the chosen position.

The paper is organized as follows. In Section II we fix our notation and definitions that are used throughout the paper. In Section III we find the exact capacity of end duplication. In Section IV study tandem duplication. Section $\mathrm{V}$ presents the capacity interspersed duplication. We conclude in Section VI by providing some insight and comparisons with the combinatorial capacity and Pólya urn models.

\section{PRELIMINARIES}

Let $\Sigma \triangleq\{0,1\}$ be the binary alphabet. The elements of $\Sigma$ are referred to as letters (symbols). While the results we present have a greater generality, for the sake of simplicity of presentation we restrict ourselves to the binary case only. We use the notation common to formal languages to describe strings over $\Sigma$. The set of length- $n$ strings (sequences) over $\Sigma$ is denoted by $\Sigma^{n}$. We let $\Sigma^{*}$ denote the set of all finitelength strings over $\Sigma$. The unique empty string is denoted by $\varepsilon$. The set of all finite-length non-empty strings is denoted by $\Sigma^{+} \triangleq \Sigma^{*} \backslash\{\varepsilon\}$.

To help with readability, we shall use the first lowercase letters of the roman alphabet, e.g., $a, b, c, \ldots$, to denote single letters from the alphabet $\Sigma$. We shall use the last lowercase letters of the roman alphabet, e.g., $u, v, w, \ldots$, to denote strings from $\Sigma^{*}$.

Let $w \in \Sigma^{*}$ be a string. We use $|w|$ to denote the length of $w$, i.e., the number of letters it contains. Obviously, $|\varepsilon|=0$. If $w^{\prime} \in \Sigma^{*}$, the concatenation of $w$ and $w^{\prime}$ is denoted $w w^{\prime}$. For $i \in \mathbb{N}$, the $i$ th letter of a string $w \in \Sigma^{*}$ (assuming $|w| \geqslant i$ ) will be denoted by $w_{i}$, i.e., $w=w_{1} w_{2} \ldots w_{|w|}$ with $w_{j} \in \Sigma$ for all $j$.

The number of occurrences of a symbol $a \in \Sigma$ in the string $w$ is denoted by $|w|_{a}$. If $w \neq \varepsilon$, then the frequency of $a \in \Sigma$ in $w$ is defined by $\mathrm{fr}_{a}(w) \triangleq|w|_{a} /|w|$.

For a natural number $n \in \mathbb{N}$ we use $[n]$ to denote the set $[n] \triangleq\{1,2, \ldots, n\}$. We also recall the definition of the binary entropy function, $H_{2}:[0,1] \rightarrow[0,1]$ defined as

$$
H_{2}(x)=-x \log _{2}(x)-(1-x) \log _{2}(1-x) .
$$

Example 1. Let $w=0011$ and $w^{\prime}=001$. We have that $w_{4}=$ $1, w w^{\prime}=0011001$ with $|w|_{0}=2$ and $\left|w w^{\prime}\right|_{0}=4$. Also, $\operatorname{fr}_{0}(w)=1 / 2$ while $\mathrm{fr}_{0}\left(w w^{\prime}\right)=4 / 7$.
The Pólya string model may be quite generally defined. Intuitively, the model takes a starting string, and in a sequence of steps, mutates it over time. A formal definition follows.

Definition 2. A Pólya string model is defined by $S=(\Sigma, s, T)$, where $\Sigma$ is a finite alphabet, $S(0)=s \in \Sigma^{+}$is a seed string, and $T: \Sigma^{*} \rightarrow \Sigma^{*}$ is a non-deterministic duplication rule. The string model is the following discrete-time random process: For all $i \in \mathbb{N}$ set $S(i)=T(S(i-1))$.

Several rule choices parallel the combinatorial (deterministic) systems studied in [6], and are special cases of the general stochastic systems studied in [5]. In particular, we define the following three Pólya string models, which we study in the rest of the paper. All three models share the fact that the mutation rule chooses a random location in the string it is given, and duplicates the single symbol appearing in that location. The duplicate symbol however is noisy, namely, it may be seen as if having passed through a binary asymmetric channel. The rules differ in the location the new symbol is inserted. The three models are defined as follows:

a) End Duplication: For any real numbers $\delta_{0}, \delta_{1} \in$ $[0,1]$, the end-duplication system is defined as $S_{\delta_{0}, \delta_{1}}^{\text {end }}=$ $\left(\Sigma, s, T_{\delta_{0}, \delta_{1}}^{\text {end }}\right)$, where for all $w \in \Sigma^{+}$,

$$
T_{\delta_{0}, \delta_{1}}^{\mathrm{end}}(w) \triangleq u a v b
$$

Here $u, v \in \Sigma^{*}, a, b \in \Sigma$, uav $=w$, the length $|u a|$ is chosen randomly independently and uniformly from $[|w|]$, and $\operatorname{Pr}(a=b \mid a=i)=1-\delta_{i}$. In essence, this nondeterministic rule chooses a uniformly random position in $w$, and duplicates the letter there to the end of the word. If the chosen bit is $a=0$, the duplicated symbol is complemented with probability $\delta_{0}$, and similarly, if $a=1$ the duplicated bit is complemented with probability $\delta_{1}$.

b) Tandem Duplication: Similarly, for any real numbers $\delta_{0}, \delta_{1} \in[0,1]$, the tandem-duplication system is defined as $S_{\delta_{0}, \delta_{1}}^{\tan }=\left(\Sigma, s, T_{\delta_{0}, \delta_{1}}^{\tan }\right)$, where for all $w \in \Sigma^{+}$,

$$
T_{\delta_{0}, \delta_{1}}^{\tan }(w) \triangleq u a b v
$$

Here $u, v \in \Sigma^{*}, a, b \in \Sigma$, uav $=w$, the length $|u a|$ is chosen randomly independently and uniformly from $[|w|]$, and $\operatorname{Pr}(a=b \mid a=i)=1-\delta_{i}$. This time, the $T_{\delta_{0}, \delta_{1}}^{\tan }$ rule chooses a uniformly random position in $w$, and duplicates the letter there right after its original position. If the chosen bit is $a=0$, the duplicated symbol is complemented with probability $\delta_{0}$, and similarly, if $a=1$ the duplicated bit is complemented with probability $\delta_{1}$.

c) Interspersed Duplication: Finally, for any real numbers $\delta_{0}, \delta_{1} \in[0,1]$, the interspersed-duplication system is defined as $S_{\delta_{0}, \delta_{1}}^{\text {int }}=\left(\Sigma, s, T_{\delta_{0}, \delta_{1}}^{\text {int }}\right)$, where for all $w \in \Sigma^{+}$,

$$
T_{\delta_{0}, \delta_{1}}^{\text {int }}(w) \triangleq u^{\prime} b v^{\prime} \text {. }
$$

Here $u, v, u^{\prime}, v^{\prime} \in \Sigma^{*}, a, b \in \Sigma, u a v=w=u^{\prime} v^{\prime}$. The length $|u a|$ is chosen randomly independently and uniformly from $[|w|]$. Additionally, the length $\left|u^{\prime} b\right|$ is also chosen randomly independently and uniformly from $[|w|+1]$. As for the inserted letter $b, \operatorname{Pr}(a=b \mid a=i)=1-\delta_{i}$. Intuitively, the $T_{\delta_{0}, \delta_{1}}^{\mathrm{int}}$ rule chooses a uniformly random position in $w$, and 


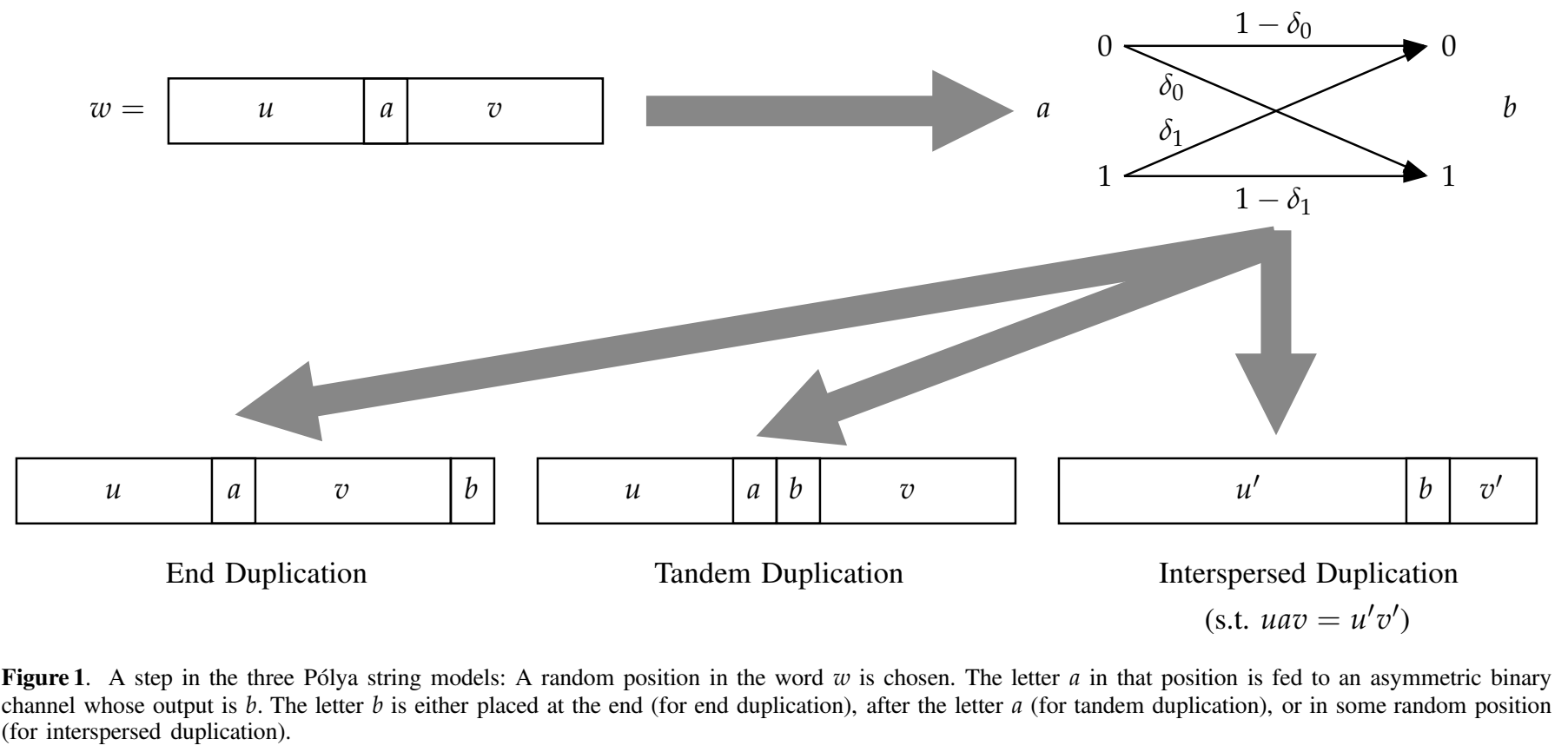
channel whose output is $b$. The letter $b$ is either placed at the end (for end duplication), after the letter $a$ (for tandem duplication), or in some random position (for interspersed duplication).

duplicates the letter there to a uniformly chosen position. Like before, if the chosen bit is $a=0$, the duplicated symbol is complemented with probability $\delta_{0}$, and similarly, if $a=1$ the duplicated bit is complemented with probability $\delta_{1}$.

A step in each of the three Pólya string systems described above is depicted in Figure 1

Given a Pólya string system $S$, the set of choices leading from $S(0)$ to $S(n)$ is denoted by $\mathcal{H}(n)$ and is referred to as the history of the sequence. The capacity of the process $S$ is defined as

$$
\operatorname{cap}(S) \triangleq \limsup _{n \rightarrow \infty} \frac{1}{n} H(S(n)),
$$

where $H$ is the entropy function,

$$
H(S(n)) \triangleq-\sum_{w \in \Sigma^{*}} \operatorname{Pr}(S(n)=w) \log _{2} \operatorname{Pr}(S(n)=w) .
$$

Since $H(S(n) \mid \mathcal{H}(n))=0$,

$$
\operatorname{cap}(S)=\limsup _{n \rightarrow \infty} \frac{1}{n} I(S(n) ; \mathcal{H}(n)),
$$

where $I$ denotes mutual information. Thus $\operatorname{cap}(S)$ can be viewed as the capacity of the channel that transforms histories to sequences and can be used to derive rate-distortion results on estimating the history $\mathcal{H}(n)$ using the sequence $S(n)$.

\section{END DUPLICATION}

We start our exploration of Pólya string systems with the end-duplication system. We distinguish between two cases that require different treatment. We first study the end-duplication system where the duplicated bit is unchanged (i.e., never complemented).

\section{A. The Noiseless Channel: $\delta_{0}=\delta_{1}=0$}

Theorem 3. Let $\Sigma=\{0,1\}, s \in \Sigma^{+}$be a seed string, and denote $t_{0} \triangleq|s|_{0}, t_{1} \triangleq|s|_{1}$. If $t_{0}, t_{1} \geqslant 1$, then the capacity of $S=S_{0,0}^{\text {end }}=\left(\Sigma, s, T_{0,0}^{\text {end }}\right)$ is

$$
\begin{aligned}
\operatorname{cap}\left(S_{0,0}^{\text {end }}\right) & =\int_{0}^{1} \beta\left(p ; t_{0}, t_{1}\right) H_{2}(p) \mathrm{d} p \\
& =\frac{\log _{2} e}{t_{0}+t_{1}}\left(\left(t_{0}+t_{1}\right) \mathrm{H}_{t_{0}+t_{1}}-t_{0} \mathrm{H}_{t_{0}}-t_{1} \mathrm{H}_{t_{1}}\right),
\end{aligned}
$$

where

$$
\beta\left(p ; t_{0}, t_{1}\right) \triangleq \frac{\left(t_{0}+t_{1}-1\right) !}{\left(t_{0}-1\right) !\left(t_{1}-1\right) !} p^{t_{0}-1}(1-p)^{t_{1}-1},
$$

is the pdf for the Beta $\left(t_{0}, t_{1}\right)$ distribution, and where $\mathrm{H}_{m}$ denotes the $m$ th harmonic number,

$$
\mathrm{H}_{m} \triangleq \sum_{i=1}^{m} \frac{1}{i}
$$

Proof: Fix any $w \in \Sigma^{n}$, and denote $k_{0} \triangleq|w|_{0}, k_{1} \triangleq|w|_{1}$, hence $k_{0}+k_{1}=n$. It is a simple exercise to show that

$$
\begin{aligned}
& \operatorname{Pr}(S(n)=s w)=f\left(t_{0}, t_{1}, k_{0}, k_{1}\right) \\
& \quad \triangleq \frac{\left(t_{0}+t_{1}-1\right) !\left(t_{0}+k_{0}-1\right) !\left(t_{1}+k_{1}-1\right) !}{\left(t_{0}-1\right) !\left(t_{1}-1\right) !\left(t_{0}+t_{1}+k_{0}+k_{1}-1\right) !} .
\end{aligned}
$$

We note that this probability does not depend on the order of bits in $w$. Thus, let us denote by $A_{k_{0}}$ the event that $S(n)=s w$, and $|w|_{0}=k_{0}$. Obviously,

$$
\operatorname{Pr}\left(A_{k_{0}}\right)=\left(\begin{array}{c}
n \\
k_{0}
\end{array}\right) f\left(t_{0}, t_{1}, k_{0}, n-k_{0}\right) .
$$


We now have,

$$
\begin{aligned}
& \operatorname{cap}(S) \\
& =\limsup _{n \rightarrow \infty} \frac{1}{n} H(S(n)) \\
& =-\limsup _{n \rightarrow \infty} \frac{1}{n} \sum_{w \in \Sigma^{n}}\left(f\left(t_{0}, t_{1},|w|_{0},|w|_{1}\right)\right. \\
& \left.\quad \cdot \log _{2} f\left(t_{0}, t_{1},|w|_{0},|w|_{1}\right)\right) \\
& =-\limsup _{n \rightarrow \infty} \frac{1}{n} \sum_{k_{0}=0}^{n} \operatorname{Pr}\left(A_{k_{0}}\right) \log _{2} f\left(t_{0}, t_{1}, k_{0}, n-k_{0}\right) \\
& =-\limsup _{n \rightarrow \infty} \frac{1}{n} \sum_{k_{0}=0}^{n}\left(\operatorname{Pr}\left(A_{k_{0}}\right)\right. \\
& \left.\quad \cdot \log _{2} \frac{\left(t_{0}+k_{0}-1\right) !\left(t_{1}+n-k_{0}-1\right) !}{\left(t_{0}+t_{1}+n-1\right) !}\right) \\
& -\frac{1}{n} \log _{2} \frac{\left(t_{0}+t_{1}-1\right) !}{\left(t_{0}-1\right) !\left(t_{1}-1\right) !}
\end{aligned}
$$

and we note that the last term is $o(1)$. We also have,

$$
\begin{aligned}
& \frac{\left(t_{0}+k_{0}-1\right) !\left(t_{1}+n-k_{0}-1\right) !}{\left(t_{0}+t_{1}+n-1\right) !} \\
& =\frac{1}{t_{0}+t_{1}+n-1}\left(\begin{array}{c}
t_{0}+t_{1}+n-2 \\
t_{0}+k_{0}-1
\end{array}\right)^{-1} .
\end{aligned}
$$

Thus,

$$
\operatorname{cap}(S)=\limsup _{n \rightarrow \infty} \frac{1}{n} \sum_{k_{0}=0}^{n} \operatorname{Pr}\left(A_{k_{0}}\right)\left(\begin{array}{c}
t_{0}+t_{1}+n-2 \\
t_{0}+k_{0}-1
\end{array}\right) .
$$

We list two more facts. The first is the well known approximation to the binomial coefficient (e.g., see [16]), giving us

$$
\left(\begin{array}{c}
t_{0}+t_{1}+n-2 \\
t_{0}+k_{0}-1
\end{array}\right)=2^{n\left(H_{2}\left(k_{0} / n\right)+o(1)\right)} .
$$

The second fact (e.g., see [17, Ch. 3]) is that for every real $p \in[0,1]$,

$$
\lim _{n \rightarrow \infty} \sum_{i=0}^{n p} \operatorname{Pr}\left(A_{i}\right)=\int_{0}^{p} \beta\left(u ; t_{0}, t_{1}\right) \mathrm{d} u .
$$

Putting this all together we obtain

$$
\operatorname{cap}(S)=\int_{0}^{1} \beta\left(p ; t_{0}, t_{1}\right) H_{2}(p) \mathrm{d} p,
$$

thus, proving the first claim.

We continue to prove the second claim. Consider the following integral:

$$
\int_{0}^{1} p^{t_{0}+\epsilon}(1-p)^{t_{1}-1} \mathrm{~d} p
$$

We use a Taylor series to obtain,

$$
p^{\epsilon}=2^{\epsilon \log _{2} p}=\sum_{i=0}^{\infty} \frac{\epsilon^{i}(\ln 2)^{i}}{i !}\left(\log _{2} p\right)^{i} .
$$

Plugging this in (3) we get

$$
\begin{aligned}
\int_{0}^{1} p^{t_{0}+\epsilon}(1-p)^{t_{1}-1} \mathrm{~d} p \\
=\sum_{i=0}^{\infty} \frac{\epsilon^{i}(\ln 2)^{i}}{i !} \int_{0}^{1} p^{t_{0}}(1-p)^{t_{1}-1}\left(\log _{2} p\right)^{i} \mathrm{~d} p .
\end{aligned}
$$

We recall the definition of the gamma function (e.g., see [11, Ch. 11]),

$$
\Gamma(x) \triangleq \int_{0}^{\infty} t^{x-1} e^{-t} \mathrm{~d} t .
$$

Additionally, $\Gamma(x+1)=x \Gamma(x)$, and in particular, for all $m \in \mathbb{N}, \Gamma(m+1)=m !$. We also recall the beta function,

$$
B(x, y) \triangleq \int_{0}^{1} t^{x-1}(1-t)^{y-1} \mathrm{~d} t=\frac{\Gamma(x) \Gamma(y)}{\Gamma(x+y)},
$$

for all $x, y \in \mathbb{R}, x, y>0$. Thus, (3) becomes

$$
\begin{aligned}
\int_{0}^{1} p^{t_{0}+\epsilon}(1-p)^{t_{1}-1} \mathrm{~d} p \\
=\frac{\Gamma\left(t_{0}+\epsilon+1\right) \Gamma\left(t_{1}\right)}{\Gamma\left(t_{0}+t_{1}+\epsilon+1\right)} \\
=\frac{\left(t_{1}-1\right) !}{\left(t_{0}+t_{1}+\epsilon\right)\left(t_{0}+t_{1}+\epsilon-1\right) \ldots\left(t_{0}+\epsilon+1\right)} \\
=\frac{\left(t_{1}-1\right) !}{\left(t_{0}+t_{1}\right)\left(1+\frac{\epsilon}{t_{0}+t_{1}}\right) \ldots\left(t_{0}+1\right)\left(1+\frac{\epsilon}{t_{0}+1}\right)} \\
=\frac{\left(t_{1}-1\right) ! t_{0} !}{\left(t_{0}+t_{1}\right) !} \cdot \frac{1}{\left(1+\frac{\epsilon}{t_{0}+t_{1}}\right) \ldots\left(1+\frac{\epsilon}{t_{0}+1}\right)} .
\end{aligned}
$$

Using a Taylor series,

Hence,

$$
e^{\frac{\epsilon}{t_{0}+t_{1}}}=1+\frac{\epsilon}{t_{0}+t_{1}}+O\left(\epsilon^{2}\right) .
$$

$$
\begin{aligned}
\int_{0}^{1} p^{t_{0}+\epsilon}(1-p)^{t_{1}-1} \mathrm{~d} p \\
=\frac{\left(t_{1}-1\right) ! t_{0} !}{\left(t_{0}+t_{1}\right) !} \cdot e^{-\left(\frac{1}{t_{0}+t_{1}}+\cdots+\frac{1}{t_{0}+1}\right) \epsilon}+O\left(\epsilon^{2}\right) \\
=\frac{\left(t_{1}-1\right) ! t_{0} !}{\left(t_{0}+t_{1}\right) !} \cdot e^{-\left(\mathrm{H}_{t_{0}+t_{1}}-\mathrm{H}_{t_{0}}\right) \epsilon}+O\left(\epsilon^{2}\right) .
\end{aligned}
$$

Yet another Taylor series we get

$$
e^{-\left(\mathrm{H}_{t_{0}+t_{1}}-\mathrm{H}_{t_{0}}\right) \epsilon}=1-\left(\mathrm{H}_{t_{0}+t_{1}}-\mathrm{H}_{t_{0}}\right) \epsilon+O\left(\epsilon^{2}\right) .
$$

Plugging this back, we obtain

$$
\begin{aligned}
& \int_{0}^{1} p^{t_{0}+\epsilon}(1-p)^{t_{1}-1} \mathrm{~d} p \\
& \quad=\frac{\left(t_{1}-1\right) ! t_{0} !}{\left(t_{0}+t_{1}\right) !}\left(1-\left(\mathrm{H}_{t_{0}+t_{1}}-\mathrm{H}_{t_{0}}\right) \epsilon\right)+O\left(\epsilon^{2}\right) .
\end{aligned}
$$

By equating the coefficient of $\epsilon^{1}$ in (4) and (5) we get

$$
\begin{aligned}
& \frac{\ln 2}{1 !} \int_{0}^{1} p^{t_{0}}(1-p)^{t_{1}-1}\left(\log _{2} p\right) \mathrm{d} p \\
& \quad=-\frac{\left(t_{1}-1\right) ! t_{0} !}{\left(t_{0}+t_{1}\right) !}\left(\mathrm{H}_{t_{0}+t_{1}}-\mathrm{H}_{t_{0}}\right) .
\end{aligned}
$$

We now repeat the same process, but instead of starting with (3), we take

$$
\int_{0}^{1} p^{t_{0}-1}(1-p)^{t_{1}+\epsilon} \mathrm{d} p
$$

and we get

$$
\begin{gathered}
\frac{\ln 2}{1 !} \int_{0}^{1} p^{t_{0}-1}(1-p)^{t_{1}}\left(\log _{2}(1-p)\right) \mathrm{d} p \\
=-\frac{\left(t_{0}-1\right) ! t_{1} !}{\left(t_{0}+t_{1}\right) !}\left(\mathrm{H}_{t_{0}+t_{1}}-\mathrm{H}_{t_{1}}\right) .
\end{gathered}
$$


Finally,

$$
\begin{aligned}
\operatorname{cap}(S)= & \int_{0}^{1} \beta\left(p ; t_{0}, t_{1}\right) H_{2}(p) \mathrm{d} p \\
= & \frac{\left(t_{0}+t_{1}-1\right) !}{\left(t_{0}-1\right) !\left(t_{1}-1\right) !}\left(\int_{0}^{1} p^{t_{0}}(1-p)^{t_{1}-1}\left(\log _{2} p\right) \mathrm{d} p\right. \\
& \left.\quad+\int_{0}^{1} p^{t_{0}-1}(1-p)^{t_{1}}\left(\log _{2}(1-p)\right) \mathrm{d} p\right) \\
= & \frac{\log _{2} e}{t_{0}+t_{1}}\left(\left(t_{0}+t_{1}\right) \mathrm{H}_{t_{0}+t_{1}}-t_{0} \mathrm{H}_{t_{0}}-t_{1} \mathrm{H}_{t_{1}}\right),
\end{aligned}
$$

thus, proving the second claim as well.

We comment that the case of either $t_{0}=0$ or $t_{1}=0$ in Theorem 3 is not interesting since then we have only strings of repeated symbols, and therefore, capacity 0.

\section{B. The Noisy Channel: $\delta_{0}+\delta_{1}>0$}

We move on to the case where the duplicated bit is passed through a noisy asymmetric binary channel. Calculating the capacity explicitly is not a simple task. This is due to the fact that in contrast to the previous case of $S_{0,0}^{\text {end }}$, the probability of obtaining a specific sequence is not a function of the frequency of symbols as in (1). This is demonstrated in the following example.

Example 4. Consider $S=S_{1,1}^{\text {end }}\left(\Sigma, s, T_{1,1}^{\text {end }}\right)$, with $s=01$. Calculating the probability of the sequences $S(3)=01110$ and $S(3)=01011$ for we obtain

$$
\begin{aligned}
\operatorname{Pr}\left(S_{1,1}^{\text {end }}(3)=01110\right) & =\frac{1}{2} \cdot \frac{1}{3} \cdot \frac{3}{4} \\
& \neq \frac{1}{2} \cdot \frac{2}{3} \cdot \frac{2}{4}=\operatorname{Pr}\left(S_{1,1}^{\text {end }}(3)=01011\right) .
\end{aligned}
$$

The following lemma will be instrumental in finding the capacity of $S_{\delta_{0}, \delta_{1}}^{\text {end }}$.

Lemma 5. Let $\Sigma=\{0,1\}, s \in \Sigma^{+}$be a seed string, and denote $S=S_{\delta_{0}, \delta_{1}}^{\text {end }}=\left(\Sigma, s, T_{\delta_{0}, \delta_{1}}^{\text {end }}\right)$. If for any real $\epsilon_{1}, \epsilon_{2}>0$, there exists $N \in \mathbb{N}$ such that for all $n \geqslant N$, $\operatorname{Pr}\left(\left|\operatorname{fr}_{0}(S(n))-\alpha\right| \leqslant \epsilon_{1}\right) \geqslant 1-\epsilon_{2}$ for some real $\alpha \in[0,1]$, then

$$
\operatorname{cap}\left(S_{\delta_{0}, \delta_{1}}^{\text {end }}\right)=H_{2}\left(\alpha\left(1-\delta_{0}\right)+(1-\alpha) \delta_{1}\right) .
$$

Proof: For our convenience, let $g:[0,1] \rightarrow[0,1]$ be defined as $g(x) \triangleq x\left(1-\delta_{0}\right)+(1-x) \delta_{1}$. Fix some real $\delta>0$. Since $H_{2}(g(x))$ is continuous, by the Heine-Cantor Theorem $\mathrm{H}_{2}(g(x))$ is uniformly continuous. Thus, there exists $\epsilon_{1}>0$ such that for all $x_{1}, x_{2} \in[0,1],\left|x_{1}-x_{2}\right| \leqslant \epsilon_{1}$ implies

$$
\left|H_{2}\left(g\left(x_{1}\right)\right)-H_{2}\left(g\left(x_{2}\right)\right)\right| \leqslant \frac{1}{2} \delta .
$$

We note that for $S=S_{\delta_{0}, \delta_{1}}^{\text {end }}$, and all $w \in \Sigma^{n+|s|}$, we have

$$
\operatorname{Pr}(S(n+1)=w 0 \mid S(n)=w)=g\left(\mathrm{fr}_{0}(w)\right) .
$$

Additionally, by the theorem requirements we are assured we can find $N \in \mathbb{N}$ such that for all $n \geqslant N$ we have

$$
\operatorname{Pr}\left(\left|\operatorname{fr}_{0}(S(n))-\alpha\right| \leqslant \epsilon_{1}\right) \geqslant 1-\frac{1}{2} \delta .
$$

For the rest of the proof, we consider the underlying sample space to be the space of all infinite sequences,

$$
\Sigma^{\mathbb{N}} \triangleq\left\{a_{1} a_{2} a_{3} \ldots: \quad \forall i \in \mathbb{N}, a_{i} \in \Sigma\right\} .
$$

A distribution $\mu$ on $\Sigma^{\mathbb{N}}$ is induced by evolving from the seed $s$ according to $S$. Thus, $S(n)$ is a random variable taking values from $\Sigma^{|s|+n}$, whose distribution is the marginal of $\mu$ on the first $|s|+n$ coordinates (sometimes called the $(|s|+n)$-length cylinder). Namely, the event $S(n)=w$ is the set

$$
\left\{v \in \Sigma^{\mathbb{N}}: v_{i}=w_{i} \text { for all } i \in[n+|s|]\right\} \text {. }
$$

Similarly, we define $S_{i}$ to be the projection of $\mu$ on the $(|s|+$ i) th coordinate, i.e., the event $S_{i}=a$ is the set

$$
\left\{v \in \Sigma^{\mathbb{N}}: v_{i+|s|}=a\right\}
$$

Let us define the event,

$$
F \triangleq\left\{v \in \Sigma^{\mathbb{N}}: \forall n \geqslant N,\left|\operatorname{fr}_{0}\left(v_{1} \ldots v_{n}\right)-\alpha\right| \leqslant \epsilon_{1}\right\},
$$

and denote by $F^{c}$ its complement. We obtain that,

$$
\begin{aligned}
\operatorname{cap}(S) & =\limsup _{n \rightarrow \infty} \frac{1}{n} H(S(n)) \\
& \leqslant \limsup _{n \rightarrow \infty} \frac{1}{n}\left(H(S(n) \mid F)+\frac{1}{2} \delta H\left(S(n) \mid F^{c}\right)\right) \\
& \leqslant \limsup _{n \rightarrow \infty} \frac{1}{n} H(S(n) \mid F)+\frac{1}{2} \delta \\
& \stackrel{(a)}{=} \limsup _{n \rightarrow \infty} \frac{1}{n} \sum_{i=1}^{n} H\left(S_{i} \mid S(i-1), F\right)+\frac{1}{2} \delta \\
& \stackrel{(b)}{\leqslant} \limsup _{n \rightarrow \infty} \frac{1}{n}\left(\sum_{i=1}^{N} H\left(S_{i}\right)\right. \\
& \left.+\sum_{i=N+1}^{n} H\left(S_{i} \mid S(i-1), F\right)\right)+\frac{1}{2} \delta \\
& \leqslant \limsup _{n \rightarrow \infty} \frac{1}{n}\left(N+(n-N)\left(H_{2}(g(\alpha))+\frac{\delta}{2}\right)\right)+\frac{1}{2} \delta \\
& =H_{2}(g(\alpha))+\delta
\end{aligned}
$$

where $(a)$ follows from the chain rule for entropy, $(b)$ follows since conditioning reduces entropy, and $(c)$ follows since

$$
H\left(S_{i} \mid S(i-1), F\right)=H_{2}\left(g\left(\operatorname{fr}_{0}(S(i-1))\right)\right)
$$

and from (6).

Using similar reasoning,

$$
\begin{aligned}
\operatorname{cap}(S) & =\limsup _{n \rightarrow \infty} \frac{1}{n} H(S(n)) \\
& \geqslant \limsup _{n \rightarrow \infty} \frac{1}{n}\left(1-\frac{1}{2} \delta\right) H(S(n) \mid F) \\
& =\limsup _{n \rightarrow \infty} \frac{1}{n}\left(1-\frac{1}{2} \delta\right) \sum_{i=1}^{n} H\left(S_{i} \mid S(i-1), F\right) \\
& \geqslant \limsup _{n \rightarrow \infty} \frac{1}{n}\left(1-\frac{1}{2} \delta\right) \sum_{i=N+1}^{n} H\left(S_{i} \mid S(i-1), F\right) \\
& \geqslant \limsup _{n \rightarrow \infty} \frac{1}{n}\left(1-\frac{1}{2} \delta\right)(n-N)\left(H_{2}(g(\alpha))-\frac{\delta}{2}\right)
\end{aligned}
$$




$$
\begin{aligned}
& =\left(1-\frac{1}{2} \delta\right)\left(H_{2}(g(\alpha))-\frac{\delta}{2}\right) \\
& \geqslant H_{2}(g(\alpha))-\delta,
\end{aligned}
$$

where the last inequality follows from the fact that $\frac{1}{2} \delta(H(\alpha)-$ $\left.\frac{\delta}{2}\right) \leqslant \frac{1}{2} \delta$.

We now have

$$
H_{2}(g(\alpha))-\delta \leqslant \operatorname{cap}(S) \leqslant H_{2}(g(\alpha))-\delta .
$$

Taking the limit as $\delta \rightarrow 0^{+}$gives the claimed result.

The next step in finding the capacity of $S_{\delta_{0}, \delta_{1}}^{\text {end }}$ is to find the (almost sure) limit of the frequency of symbols. We make use of the following definition.

Definition 6. Let $\left(x_{n}\right)_{n \in \mathbb{N}}$ be a sequence of real numbers, evolving according to the equation $x_{n+1}=x_{n}+a \cdot f\left(x_{n}\right)$ for some function $f: \mathbb{R} \rightarrow \mathbb{R}$ and a constant $a \in \mathbb{R}$, $a>0$. We say that $x^{\prime}$ is an equilibrium point of the recursion $x_{n+1}=x_{n}+a \cdot f\left(x_{n}\right)$ if $f\left(x^{\prime}\right)=0$.

We prove the next lemma using stochastic approximation (for a comprehensive study see [3]).

Lemma 7. Let $\Sigma=\{0,1\}, s \in \Sigma^{+}$be a seed string, and denote $S=S_{\delta_{0}, \delta_{1}}^{\text {end }}=\left(\Sigma, s, T_{\delta_{0}, \delta_{1}}^{\text {end }}\right)$, where $\delta_{0}+\delta_{1}>0$. Then

$$
\lim _{n \rightarrow \infty} \operatorname{fr}_{0}(S(n))=\frac{\delta_{1}}{\delta_{0}+\delta_{1}}
$$

almost surely.

Proof: Let $t_{0} \triangleq|s|_{0}$ and $t_{1} \triangleq|s|_{1}$. We further define

$$
x_{n} \triangleq|S(n)|_{0}, \quad z_{n} \triangleq \operatorname{fr}_{0}(S(n))=\frac{x_{n}+t_{0}}{n+t_{0}+t_{1}} .
$$

Let $g:[0,1] \rightarrow[0,1]$ be defined as

$$
g(x) \triangleq x\left(1-\delta_{0}\right)+(1-x) \delta_{1} .
$$

Note that for any $w \in \Sigma^{n+|s|}$,

$$
\operatorname{Pr}(S(n+1)=w 0 \mid S(n)=w)=g\left(z_{n}\right),
$$

and that $z_{0}=\frac{t_{0}}{t_{0}+t_{1}}$. We write

$$
x_{n+1}=x_{n}+\xi_{n+1}
$$

where $\xi_{n+1}=1$ if the $(n+1)$ st appended symbol (due to mutation) is a 0 , and $\xi_{n+1}=0$ otherwise. A simple calculation yields

$$
\begin{aligned}
z_{n+1} & =z_{n}+\frac{1}{n+1+t_{0}+t_{1}}\left(\xi_{n+1}-z_{n}\right) \\
& =z_{n}+\frac{\left(g\left(z_{n}\right)-z_{n}\right)+\left(\xi_{n+1}-g\left(z_{n}\right)\right)}{n+1+t_{0}+t_{1}} .
\end{aligned}
$$

The main goal is to find the limit points of the sequence $z_{n}$.

Let $M_{n} \triangleq \xi_{n}-f\left(z_{n-1}\right)$, and note that $M_{n}$ is a martingale difference sequence. Indeed, if $\mathcal{F}_{n}$ is the $\sigma$-algebra generated by $\sigma\left(z_{m}, M_{m}, m \leqslant n\right)$ then

$$
\begin{aligned}
E\left[M_{n+1} \mid \mathcal{F}_{n}\right] & =E\left[\xi_{n+1} \mid \mathcal{F}_{n}\right]-g\left(z_{n}\right) \\
& =g\left(z_{n}\right)-g\left(z_{n}\right) \\
& =0 .
\end{aligned}
$$

Hence, the limiting differential equation $z_{n}$ is expected to track is given by

$$
\dot{z}_{t}=g\left(z_{t}\right)-z_{t}
$$

In order for the differential equation to have a unique solution for any $z_{0}$, we need to show that $g(z)-z$ is Lipschitz [3, Ch. 11, Theorem 5]. Indeed,

$$
|(g(z)-z)-(g(y)-y)|=\left|\left(\delta_{0}+\delta_{1}\right)(z-y)\right|,
$$

which means that $g(z)-z$ is $\left(\delta_{0}+\delta_{1}\right)$-Lipschitz. Solving the differential equation we obtain the solution

$$
z_{t}=\frac{\delta_{1}}{\delta_{0}+\delta_{1}}+\left(\frac{t_{0}}{t_{0}+t_{1}}-\frac{\delta_{1}}{\delta_{0}+\delta_{1}}\right) e^{-t\left(\delta_{0}+\delta_{1}\right)} .
$$

From the solution of the differential equation, it is clear that the set $[0,1]$ is an invariant set (any trajectory starting at $[0,1]$ and evolves according to $z_{t}$ will remain in the set). Also, we see that the point $z^{*} \triangleq \frac{\delta_{1}}{\delta_{0}+\delta_{1}}$ is an equilibrium point and since $g(z)$ is contraction (i.e., $\left|g\left(z_{1}\right)-g\left(z_{2}\right)\right| \leqslant\left|z_{1}-z_{2}\right|$ ) it has only one equilibrium point (this is due to the Banach fixed-point theorem [2]). Hence, using [3, Corollary 4], $z_{n}$ converges almost surely to $z^{*}$.

We remark that for $\delta_{0}=\delta_{1}=0$, we obtain in (7) that $\dot{z}_{t}=0$, which means that there is no singular attraction point (there is no stable equilibrium point). Hence, in order to use the same method, we need to evaluate the probability of every possible limiting point. This, as we know from the formula for $\operatorname{cap}\left(S_{0,0}^{\text {end }}\right)$ from Theorem 3, is a function of the seed string, and is related to the beta distribution.

We can now state the capacity for $S_{\delta_{0}, \delta_{1}}^{\text {end }}$ with $\delta_{0}+\delta_{1}>0$.

Theorem 8. Let $\Sigma=\{0,1\}, s \in \Sigma^{+}$be a seed string, and denote $S=S_{\delta_{0}, \delta_{1}}^{\text {end }}=\left(\Sigma, s, T_{\delta_{0}, \delta_{1}}^{\text {end }}\right)$, where $\delta_{0}+\delta_{1}>0$. Then

$$
\operatorname{cap}\left(S_{\delta_{0}, \delta_{1}}^{\text {end }}\right)=H_{2}\left(\frac{\delta_{1}}{\delta_{0}+\delta_{1}}\right)=H_{2}\left(\frac{\delta_{0}}{\delta_{0}+\delta_{1}}\right) .
$$

Proof: By Lemma 7 we obtain the limiting frequencies of $S(n)$. Then, by using Lemma 5 we obtain the desired result.

Figure 2 shows a contour plot of the capacity of $S_{\delta_{0}, \delta_{1}}^{\text {end }}$.

\section{TANDEM DUPLICATION}

We turn our attention in this section to tandem-duplication Pólya string models. We again consider several cases separately, depending on the parameters of the binary asymmetric channel, $\delta_{0}$ and $\delta_{1}$. We find the exact capacity of $S_{0,0}^{\text {tan }}$, and relate the capacity of $S_{1,1}^{\tan }$ to a combinatorial property of permutations. Finally, we upper bound the capacity of the general $S_{\delta_{0}, \delta_{1}}^{\tan }$.

\footnotetext{
${ }^{1}$ Note that [3] Corollary 4] uses the notion of internally chain transitive. In our case, since $z^{*}$ is a unique equilibrium point we obtain that the singleton $\left\{z^{*}\right\}$ is the internally chain transitive set in $[0,1]$.
} 


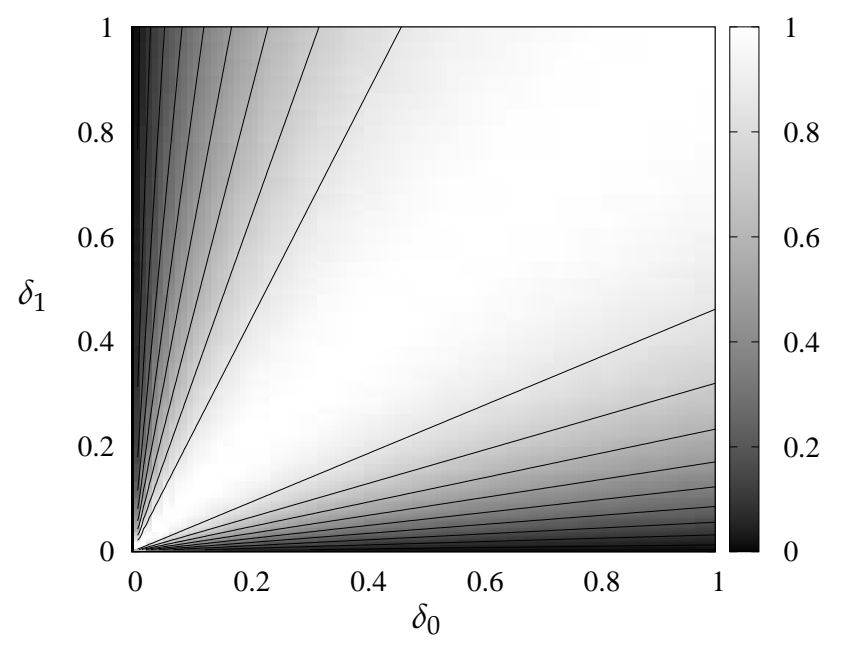

Figure 2. A contour plot of $\operatorname{cap}\left(S_{\delta_{0}, \delta_{1}}^{\text {end }}\right)$.

A. The Noiseless Channel: $\delta_{0}=\delta_{1}=0$

The capacity of the noiseless case is simple.

Theorem 9. Let $\Sigma=\{0,1\}, s \in \Sigma^{+}$be a seed string, and denote $S=S_{0,0}^{\tan }=\left(\Sigma, s, T_{0,0}^{\tan }\right)$. Then

$$
\operatorname{cap}\left(S_{0,0}^{\tan }\right)=0 .
$$

Proof: A crude counting argument suffices for the proof. Consider the initial string $S(0)$, and denote the number of runs in it by $r$. Obviously any tandem-duplication operation extends existing runs and never creates new runs. Thus, obtaining $S(n)$ may be viewed as an action of throwing $n$ balls into $r$ bins. The total number of resulting strings (regardless of probability) is given exactly by $\left(\begin{array}{c}n+r-1 \\ r-1\end{array}\right) \leqslant(n+r-1)^{r-1}$. Maximum entropy will be attained by a uniform distribution over those strings, and even in that case we get

$$
\operatorname{cap}\left(S^{\tan }\right) \leqslant \limsup _{n \rightarrow \infty} \frac{1}{n} \log _{2}(n+r-1)^{r-1}=0 .
$$

A lower bound of 0 is trivial since we have at least one string for each length $n \geqslant|s|$.

\section{B. The Complementing Channel: $\delta_{0}=\delta_{1}=1$}

Next, we consider $S_{1,1}^{\tan }$, where the duplicated bit is always complemented. For simplicity, in what follows we assume that the seed string is $S(0)=s=0$. We note then that $S(1)=01$ always. As an example, a possible history leading to $S(3)=$ 0110 is

$$
0 \rightarrow 01 \rightarrow 010 \rightarrow 0110,
$$

where in each step the new symbol is in bold.

The history of $S(n)$ can be encoded as a permutation of length $n$, called its history permutation, as follows: Replace each 0 or 1 with the number of the turn in which they were added to the sequence. For example, the history given in (8) corresponds to the history permutation 312 :

$$
\begin{aligned}
& 0 \rightarrow 01 \rightarrow 010 \rightarrow 0110 \\
& \varepsilon \rightarrow 1 \rightarrow 12 \rightarrow 312 .
\end{aligned}
$$

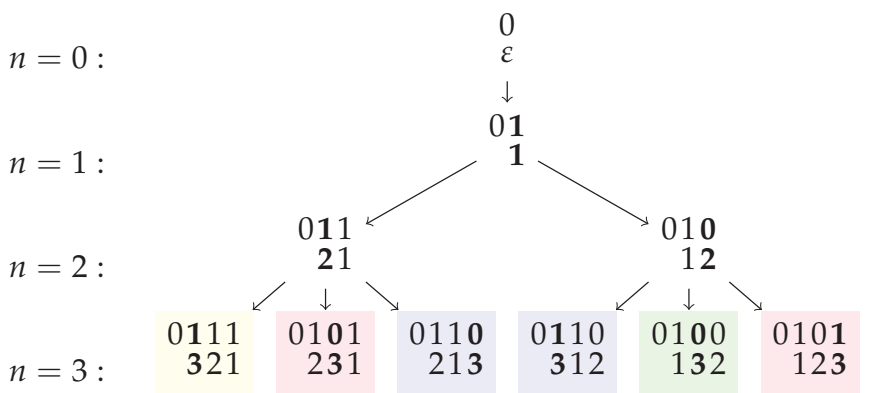

Figure 3. The tree of sequences that can be obtained starting from $s=0$ using the $T_{11}^{\tan }$ rule for $n \leqslant 3$. The first line in each node is the sequence and the second line is its history permutation.

Note that since 0 is always in the starting position, we drop it to obtain a permutation of $[n]$. It is clear that this provides us with a bijection between permutations of $[n]$ and a history resulting in a sequence $S(n)=01 w, w \in\{0,1\}^{n-1}$. This bijection will be useful in what follows.

The tree in Fig. 3 illustrates the history permutations and the sequences arising from them for $n \leqslant 3$. Since all histories are equally likely, all leaves at the same level in the tree are equally likely. Note however that not all sequences are equally likely as multiple histories may lead to the same sequence. For example, from Fig. 3, it is clear that $\operatorname{Pr}(S(3)=0101)=$ $2 \cdot \operatorname{Pr}(S(3)=0100)$.

The following definitions will be useful. For $n \in \mathbb{N}$ let $\mathbb{S}_{n}$ denote the symmetric group of permutations over $[n]$. Recall that the $i$ th letter of $S(n)$, for $i \in[n+1]$, is denoted by $S_{i}(n)$. Furthermore, if $w \in \Sigma^{*}$, and $1 \leqslant i \leqslant j \leqslant|w|$, then we denote $w_{i}^{j} \triangleq w_{i} w_{i+1} \ldots w_{j}$. For $S(n)$, this notation becomes $S_{i}^{j}(n)$.

For a permutation $\pi \in \mathbb{S}_{n}$, define its signature $\operatorname{sig}(\pi)=$ $u \in\{0,1\}^{n-1}$ such that

$$
u_{i} \triangleq \begin{cases}0, & \text { if } \pi_{i}>\pi_{i+1}, \\ 1, & \text { if } \pi_{i}<\pi_{i+1},\end{cases}
$$

for $i \in[n-1]$, i.e., ascents are marked by 1 and descents by 0 . We also define, for each $u \in\{0,1\}^{n-1}$,

$$
\Psi_{u} \triangleq\left\{\pi \in \mathrm{S}_{n}: \operatorname{sig}(\pi)=u\right\} .
$$

The following lemma is useful in computing the capacity of the system.

Lemma 10. Let $\Sigma=\{0,1\}$, and denote $S=S_{1,1}^{\text {tan }}=(\Sigma, s=$ $\left.0, T_{1,1}^{\mathrm{tan}}\right)$. Then for all $u \in \Sigma^{n-1}$,

$$
\operatorname{Pr}(S(n)=01 u)=\frac{\left|\Psi_{u}\right|}{n !} .
$$

Proof: Let the set of history permutations in $S$ that lead to $01 w$ be denoted by $\Pi_{01 w}$. For technical reasons, we will need to consider also $S^{\prime}=\left(\Sigma, s=1, T_{1,1}^{\text {tan }}\right)$ (which differs from $S$ by starting with the seed string 1 instead of 0 ). Obviously $S$ and $S^{\prime}$ are isomorphic, by simply complementing all bits. Similarly, we denote the set of history permutations in $S^{\prime}$ that lead to $10 w$ by $\Pi_{10 w}$.

To prove the claim, it suffices to show that for all $w \in \Sigma^{*}$,

$$
\left|\Pi_{01 w}\right|=\left|\Pi_{10 w}\right|=\left|\Psi_{w w}\right| .
$$


We show this by proving that the sizes of all sets satisfy the same recursion with the same initial values. The initial conditions for all recursions are

$$
\left|\Pi_{01 \varepsilon}\right|=\left|\Pi_{10 \varepsilon}\right|=\left|\Psi_{\varepsilon}\right|=1,
$$

where $\varepsilon$ is the empty string.

We start by providing two recursions for $\left|\Psi_{w}\right|$. For $v \in \Sigma^{n}$, let

$$
\begin{aligned}
T_{v} \triangleq\{i \in[n+1]: & \left(v_{i-1}=1 \text { or } i=1\right) \\
& \text { and } \left.\left(v_{i}=0 \text { or } i=n+1\right)\right\}, \\
U_{v} \triangleq\{i \in[n+1]: & \left(v_{i-1}=0 \text { or } i=1\right) \\
& \text { and } \left.\left(v_{i}=1 \text { or } i=n+1\right)\right\},
\end{aligned}
$$

be the set of positions where 1 to 0 and 0 to 1 transitions occur (except at the boundaries). For example for $v=0011010$, we have $T_{v}=\{1,5,7\}$ and $U_{v}=\{3,6,8\}$.

For $u \in \Sigma^{n}$, we can construct a permutation of $[n+1]$ with the signature $u$ recursively by first determining the position of $n+1$. The set of valid positions for $n+1$ is precisely the set $T_{u}$. Suppose we place $n+1$ in position $i \in T_{u}$. We now need to construct two permutations with signatures $u_{1}^{i-2}$ and $u_{i+1}^{n}$, each with a subset of $[n]$. We can choose the set of elements for each of these two permutations in $\left(\begin{array}{c}n \\ i-1\end{array}\right)$ ways. Hence,

$$
\left|\Psi_{u}\right|=\sum_{i \in T_{u}}\left(\begin{array}{c}
n \\
i-1
\end{array}\right)\left|\Psi_{u_{1}^{i-2}}\right|\left|\Psi_{u_{i+1}^{n}}\right|
$$

Similarly, by deciding where to place 1 (instead of $n+1$ ), we can show that

$$
\left|\Psi_{u}\right|=\sum_{i \in U_{u}}\left(\begin{array}{c}
n \\
i-1
\end{array}\right)\left|\Psi_{u_{1}^{i-2}}\right|\left|\Psi_{u_{i+1}^{n}}\right| .
$$

We now return to $\Pi_{01 u}$ and $\Pi_{10 u}$. Note that (9) holds trivially if $u$ is the empty string. Suppose (9) holds for all $u \in \Sigma^{n-1}$. Fix $u \in \Sigma^{n}$ and consider the sequence $01 u$ as the result of the Pólya string model. In the permutations in $\Pi_{01 u}$, the set of valid positions for 1 is precisely the set of positions in $T_{u}$. To see this note that in a permutation describing the history of $01 u$, the element 1 can only correspond to the last element in a run of $1 \mathrm{~s}$ in the string $01 u$. Specifically, the element 1 can be placed in position 1 iff $u$ starts with a 0 (since the bold 1 in $01 u$ is the last 1 in a run); 1 can be placed in position $2 \leqslant i \leqslant n$ iff $u_{i-1} u_{i}=10$; and finally, 1 can be placed in position $n+1$ iff $u_{n}=1$ (again, the last 1 in a run of $1 \mathrm{~s}$ ).

Hence, we can construct these permutations recursively by first determining the position of 1 in them, and

$$
\begin{aligned}
\left|\Pi_{01 u}\right| & =\sum_{i \in T_{u}}\left(\begin{array}{c}
n \\
i-1
\end{array}\right)\left|\Pi_{01 u_{1}^{i-2}}\right|\left|\Pi_{10 u_{i+1}^{n}}\right| \\
& =\sum_{i \in T_{u}}\left(\begin{array}{c}
n \\
i-1
\end{array}\right)\left|\Psi_{u_{1}^{i-2}}\right|\left|\Psi_{u_{i+1}^{n}}\right| \cdot
\end{aligned}
$$

Similarly, for $\Pi_{10 u}, u \in \Sigma^{n}$, the possible positions for 1 are precisely those in $U_{u}$ as now 1 in the history permutation should correspond to the last 0 in a run of $0 \mathrm{~s}$ in the string $10 u$. So 1 can be placed in position 1 iff $u$ starts with a 1 ; it can be placed in position $2 \leqslant i \leqslant n$ iff $u_{i-1} u_{i}=01$; and finally it can be placed in position $n+1$ if $s_{u}=0$. We thus have

$$
\begin{aligned}
\left|\Pi_{10 u}\right| & =\sum_{i \in T_{u}}\left(\begin{array}{c}
n \\
i-1
\end{array}\right)\left|\Pi_{10 u_{1}^{i-2}}\right|\left|\Pi_{01 u_{i+1}^{n}}\right| \\
& =\sum_{i \in T_{u}}\left(\begin{array}{c}
n \\
i-1
\end{array}\right)\left|\Psi_{u_{1}^{i-2}}\right|\left|\Psi_{u_{i+1}^{n}}\right| .
\end{aligned}
$$

This completes the proof of (9) for all $u \in \Sigma^{*}$.

As Lemma 10 shows, in order to find $\operatorname{cap}\left(S_{1,1}^{\tan }\right)$ with seed string $s=0$, we need to find the asymptotics of the probability that a uniformly chosen permutation from $S_{n}$ has a given signature, as $n \rightarrow \infty$. We do not yet know how to attain this goal, and instead, use simplified versions of it to obtain bounds on the aforementioned capacity.

Theorem 11. Let $\Sigma=\{0,1\}$, and denote $S=S_{1,1}^{\tan }=(\Sigma, s=$ $\left.0, T_{1,1}^{\tan }\right)$. Then,

$$
\frac{5 \log _{2} e-2}{6} \leqslant \operatorname{cap}\left(S_{1,1}^{\tan }\right) \leqslant H_{2}\left(\frac{1}{3}\right) \text {. }
$$

Proof: Define the process $\bar{S}$ as follows. Suppose we uniformly and independently choose random reals in $[0,1]$ denoted by $X_{1}, X_{2}, \ldots$ We note that for any $i \neq j, \operatorname{Pr}\left[X_{i}=\right.$ $\left.X_{j}\right]=0$, and so with probability 1 the sequence $X_{1}, \ldots, X_{n}$ induces a uniformly chosen permutation from $S_{n}$. Let

$$
\bar{S}_{i}= \begin{cases}1, & \text { if } X_{i}<X_{i+1} \\ 0, & \text { if } X_{i}>X_{i+1}\end{cases}
$$

for $i \in \mathbb{N}$. Thus, $\bar{S}_{1} \ldots \bar{S}_{n-1}$ form the signature of a uniformly chosen permutation from $S_{n}$. It follows from Lemma 10 that for any $n$ and $u \in \Sigma^{n-1}$, we have

$$
\operatorname{Pr}(S(n)=01 u)=\operatorname{Pr}\left(\bar{S}_{1}^{n-1}=u\right)
$$

Note that the strings in $S$ evolve by changing at a random position, but $\bar{S}$ can be viewed as evolving by changing at the end, and thus is easier to analyze.

We now have,

$$
\begin{aligned}
\operatorname{cap}(S) & =\limsup _{n \rightarrow \infty} \frac{1}{n} H(S(n))=\limsup _{n \rightarrow \infty} \frac{1}{n} H\left(\bar{S}_{1}^{n-1}\right) \\
& =\limsup _{n \rightarrow \infty} \frac{1}{n} \sum_{i=1}^{n-1} H\left(\bar{S}_{i} \mid \bar{S}_{1}^{i-1}\right)
\end{aligned}
$$

Before proceeding with the proof, we show a simpler lower bound than the one given in the theorem. For $i \in \mathbb{N}$, since $\bar{S}_{1}^{i-1} \rightarrow X_{i} \rightarrow S_{i}$, i.e., they form a Markov chain, we have $H\left(\bar{S}_{i} \mid \bar{S}_{1}^{i-1}\right) \geqslant H\left(\bar{S}_{i} \mid X_{i}\right)$. Furthermore, $\operatorname{Pr}\left(\bar{S}_{i}=0 \mid X_{i}=x\right)=$ $x$. Thus from (11) we find

$$
\operatorname{cap}(S) \geqslant H\left(\bar{S}_{i} \mid X_{i}\right)=\int_{0}^{1} H_{2}(x) \mathrm{d} x=\frac{\log e}{2} \geqslant 0.7213 .
$$

With the same approach we can prove the stronger lower bound in the theorem. Note that $\bar{S}_{1}^{i-2} \rightarrow X_{i-1} \rightarrow \bar{S}_{i-1}^{i}$. So

$$
\begin{aligned}
H\left(\bar{S}_{i} \mid \bar{S}_{1}^{i-1}\right) & \geqslant H\left(\bar{S}_{i} \mid \bar{S}_{i-1}, X_{i-1}\right) \\
& =\int_{0}^{1} x h_{0}(x) \mathrm{d} x+\int_{0}^{1}(1-x) h_{1}(x) \mathrm{d} x,
\end{aligned}
$$


where

$$
\begin{aligned}
& h_{0}(x)=H\left(\bar{S}_{i} \mid \bar{S}_{i-1}=0, X_{i-1}=x\right), \\
& h_{1}(x)=H\left(\bar{S}_{i} \mid \bar{S}_{i-1}=1, X_{i-1}=x\right) .
\end{aligned}
$$

We have

$$
\begin{aligned}
& h_{0}(x)=H_{2}\left(\frac{1}{x} \int_{0}^{x} y \mathrm{~d} y\right)=H_{2}\left(\frac{x}{2}\right) \\
& h_{1}(x)=H_{2}\left(\frac{1}{1-x} \int_{x}^{1}(1-y) \mathrm{d} y\right)=H_{2}\left(\frac{1-x}{2}\right) .
\end{aligned}
$$

Hence,

$$
\begin{aligned}
& H\left(\bar{S}_{i} \mid \bar{S}_{1}^{i-1}\right)=\int_{0}^{1} x H_{2}\left(\frac{x}{2}\right) \mathrm{d} x+ \\
& \quad \int_{0}^{1}(1-x) H_{2}\left(\frac{1-x}{2}\right) \mathrm{d} x=\frac{5 \log e-2}{6} \geqslant 0.8689 .
\end{aligned}
$$

Now we turn to proving the upper bound. Note that

$$
\begin{aligned}
\operatorname{cap}(S) & =\limsup _{n \rightarrow \infty} \frac{1}{n} \sum_{i=1}^{n-1} H\left(\bar{S}_{i} \mid \bar{S}_{1}^{i-1}\right) \\
& \leqslant \limsup _{n \rightarrow \infty} \frac{1}{n} \sum_{i=1}^{n-1} H\left(\bar{S}_{i} \mid \bar{S}_{i-1}\right) \\
& =H\left(\bar{S}_{2} \mid \bar{S}_{1}\right) \\
& =\frac{1}{2}\left(H\left(\bar{S}_{2} \mid \bar{S}_{1}=0\right)+H\left(\bar{S}_{2} \mid \bar{S}_{1}=1\right)\right) \\
& =\frac{1}{2} \cdot 2 \cdot H_{2}\left(\frac{1}{3}\right) \leqslant 0.9183,
\end{aligned}
$$

since by integrating over the values of $X_{1}^{3}$, we find

$$
\operatorname{Pr}\left(\bar{S}_{2}=0 \mid \bar{S}_{1}=0\right)=\frac{\int_{0}^{1} \mathrm{~d} x_{1} \int_{0}^{x_{1}} \mathrm{~d} x_{2} \int_{0}^{x_{2}} \mathrm{~d} x_{3}}{\int_{0}^{1} \mathrm{~d} x_{1} \int_{0}^{x_{1}} \mathrm{~d} x_{2}}=\frac{1 / 6}{1 / 2}=\frac{1}{3}
$$

as well as $\operatorname{Pr}\left(\bar{S}_{2}=1 \mid \bar{S}_{1}=1\right)=\frac{1}{3}$.

Both methods used in the proof of the preceding theorem can be extended to obtain better bounds, at the cost of more tedious proofs. For example, for the upper bound we can have

$$
\operatorname{cap}\left(S_{1,1}^{\tan }\right) \leqslant H\left(\bar{S}_{4} \mid \bar{S}_{2}, \bar{S}_{3}\right)
$$

Let $P_{i j k}=\operatorname{Pr}\left(\bar{S}_{2}=i, \bar{S}_{3}=j, \bar{S}_{4}=k\right)$. By integration, we find

$$
\left(P_{000}, P_{001}, \ldots, P_{111}\right)=\frac{1}{24}(1,3,5,3,3,5,3,1) .
$$

Hence

$$
\begin{aligned}
& H\left(\bar{S}_{4} \mid \bar{S}_{2}=0, \bar{S}_{3}=0\right)=H\left(\bar{S}_{4} \mid \bar{S}_{2}=1, \bar{S}_{3}=1\right)=H_{2}\left(\frac{2}{8}\right), \\
& H\left(\bar{S}_{4} \mid \bar{S}_{2}=0, \bar{S}_{3}=1\right)=H\left(\bar{S}_{4} \mid \bar{S}_{2}=1, \bar{S}_{3}=0\right)=H_{2}\left(\frac{3}{8}\right) .
\end{aligned}
$$

So

$$
\operatorname{cap}\left(S_{1,1}^{\tan }\right) \leqslant 2 \cdot \frac{1}{6} H_{2}\left(\frac{2}{8}\right)+2 \cdot \frac{1}{3} H_{2}\left(\frac{3}{8}\right) \leqslant 0.9067 .
$$

C. The Noisy Channel: $\delta_{0}+\delta_{1}>0$

Lastly, we address the general noisy case of $S_{\delta_{0}, \delta_{1}}^{\mathrm{tan}}$, with $\delta_{0}+\delta_{1}>0$. The methods used for finding the capacity of $S_{\delta_{0}, \delta_{1}}^{\text {end }}$ need to be extended: instead of studying the frequencies of letters, we shall study the frequencies of pairs of adjacent letters. To that end, we need to extend some definitions.

Let $w \in \Sigma^{n}, n \in \mathbb{N}$, and let $u \in \Sigma^{k}, k \in \mathbb{N}$, where $k \leqslant n$. The number of occurrences of $u$ in $w$ as a substring is denoted by $|w|_{u}$, formally defined as

$$
|w|_{u} \triangleq\left|\left\{i \in[n]: w_{i}^{i+n-1}=u\right\}\right|,
$$

where indices are taken cyclically, i.e., $w_{n}$ is followed by $w_{1}$. We also extend the definition of frequency,

$$
\mathrm{fr}_{u}(w) \triangleq \frac{|w|_{u}}{|w|}
$$

Lemma 12. Let $\Sigma=\{0,1\}, s \in \Sigma^{+}$a seed string, and denote $S=S_{\delta_{0}, \delta_{1}}^{\tan }=\left(\Sigma, s, T_{\delta_{0}, \delta_{1}}^{\tan }\right)$, where $\delta_{0}+\delta_{1}>0$. Then

$$
\begin{aligned}
\lim _{n \rightarrow \infty}\left(\begin{array}{c}
\operatorname{fr}_{00}(S(n)) \\
\operatorname{fr}_{01}(S(n)) \\
\operatorname{fr}_{10}(S(n)) \\
\operatorname{fr}_{11}(S(n))
\end{array}\right) \\
=\frac{1}{\left(1+\delta_{0}+\delta_{1}\right)\left(\delta_{0}+\delta_{1}\right)}\left(\begin{array}{c}
\left(1-\delta_{0}+\delta_{1}\right) \delta_{1} \\
2 \delta_{0} \delta_{1} \\
2 \delta_{0} \delta_{1} \\
\left(1-\delta_{1}+\delta_{0}\right) \delta_{0}
\end{array}\right),
\end{aligned}
$$

almost surely.

Proof: To avoid cumbersome notation, let us denote

$$
x_{n}^{u} \triangleq|S(n)|_{u^{\prime}} \quad z_{n} \triangleq\left(\begin{array}{l}
\operatorname{fr}_{00}(S(n)) \\
\operatorname{fr}_{01}(S(n)) \\
\operatorname{fr}_{10}(S(n)) \\
\operatorname{fr}_{11}(S(n))
\end{array}\right) .
$$

Let $\mathcal{F}_{n}$ be the filtration generated by $z_{n}$.

We first find the expected change in the multiplicities $x_{n+1}^{u}$ for $u \in\{00,01,10,11\}$. To do so, we need to find the number of new occurrences of 00 and the number of occurrences that are eliminated by a mutation. First, we consider $u=00$. A new occurrence of $u$ appears if 0 is duplicated or if the 1 in an occurrence of 10 is complement-duplicated (i.e., resulting in 100). An occurrence of 00 is eliminated if its first 0 is complement-duplicated. Thus

$$
\begin{aligned}
E\left[x_{n+1}^{00}-x_{n}^{00} \mid \mathcal{F}_{n}\right] & =z_{n}^{0}\left(1-\delta_{0}\right)+z_{n}^{10} \delta_{1}-z_{n}^{00} \delta_{0} \\
& =z_{n}^{00}\left(1-2 \delta_{0}\right)+z_{n}^{01}\left(1-\delta_{0}\right)+z_{n}^{10} \delta_{1} .
\end{aligned}
$$

Similarly, we have

$$
\begin{aligned}
& E\left[x_{n+1}^{01}-x_{n}^{01} \mid \mathcal{F}_{n}\right]=z_{n}^{00} \delta_{0}+z_{n}^{11} \delta_{1}, \\
& E\left[x_{n+1}^{10}-x_{n}^{10} \mid \mathcal{F}_{n}\right]=z_{n}^{00} \delta_{0}+z_{n}^{11} \delta_{1}, \\
& E\left[x_{n+1}^{11}-x_{n}^{11} \mid \mathcal{F}_{n}\right]=z_{n}^{01} \delta_{0}+z_{n}^{10}\left(1-\delta_{1}\right)+z_{n}^{11}\left(1-2 \delta_{1}\right) .
\end{aligned}
$$

By stacking these equations, we find $A^{\prime}$ such that

$$
E\left[x_{n+1}-x_{n} \mid \mathcal{F}_{n}\right]=A^{\prime} z_{n} .
$$




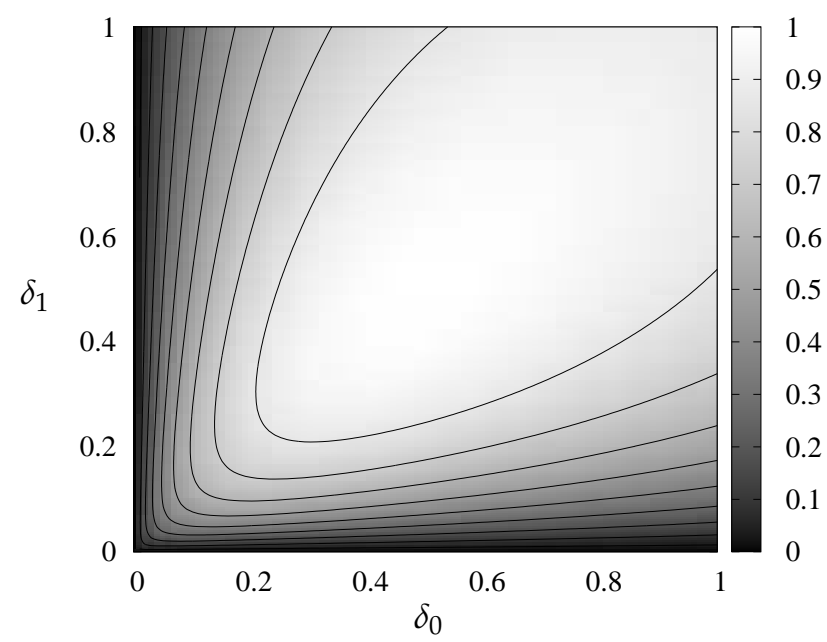

Figure 4. A contour plot of the upper bound on $\operatorname{cap}\left(S_{\delta_{0}, \delta_{1}}^{\tan }\right)$ of Theorem 13

By letting $A \triangleq A^{\prime}-I$, we find

$$
A=\left(\begin{array}{cccc}
-2 \delta_{0} & 1-\delta_{0} & \delta_{1} & 0 \\
\delta_{0} & -1 & 0 & \delta_{1} \\
\delta_{0} & 0 & -1 & \delta_{1} \\
0 & \delta_{0} & 1-\delta_{1} & -2 \delta_{1}
\end{array}\right)
$$

Using stochastic approximation, We can relate the behavior of $z_{n}$ to the ODE $\dot{z}_{t}=A z_{t}$ (see [3]). In particular, $z_{n}$ converges almost surely to the null space of $A$. From this, the theorem follows.

The capacity (entropy) of a source of strings whose limiting substring frequencies are known, was studied in [15], and an upper bound provided. We use this result to upper bound the capacity.

Theorem 13. Let $\Sigma=\{0,1\}, s \in \Sigma^{+}$a seed string, and denote $S=S_{\delta_{0}, \delta_{1}}^{\tan }=\left(\Sigma, s, T_{\delta_{0}, \delta_{1}}^{\tan }\right)$, where $\delta_{0}+\delta_{1}>0$. Then

$$
\begin{aligned}
\operatorname{cap}\left(S_{\delta_{0}, \delta_{1}}^{\tan }\right) \leqslant & \frac{\delta_{1}}{\delta_{0}+\delta_{1}} H_{2}\left(\frac{1-\delta_{0}+\delta_{1}}{1+\delta_{0}+\delta_{1}}\right) \\
& +\frac{\delta_{0}}{\delta_{0}+\delta_{1}} H_{2}\left(\frac{1-\delta_{1}+\delta_{0}}{1+\delta_{0}+\delta_{1}}\right) .
\end{aligned}
$$

Proof: Let $z_{\infty} \triangleq\left(z_{\infty}^{00}, z_{\infty}^{01}, z_{\infty}^{10}, z_{\infty}^{11}\right)^{T}$ be the limit given by Lemma[12. From [15], the capacity is upper bounded above by

$$
\operatorname{cap}(S) \leqslant-\sum_{u_{1} u_{2}} z_{\infty}^{u_{1} u_{2}} \log \frac{z_{\infty}^{u_{1} u_{2}}}{z_{\infty}^{u_{1} u_{2}}+z_{\infty}^{u_{1} \bar{u}_{2}}},
$$

where $u_{1}, u_{2} \in\{0,1\}$ and $\bar{u}_{i}=1-u_{i}$. From this, by substituting the expression for $z_{\infty}$ given in Lemma 12, the claim follows.

The upper bound on the capacity of $S_{\delta_{0}, \delta_{1}}^{\tan }$ is shown in a contour plot in Figure 4

We briefly discuss two extreme cases. For $\delta_{0}=\delta_{1}=$ $\frac{1}{2}$, the upper bound states that $\operatorname{cap}\left(S_{1 / 2,1 / 2}^{\tan }\right) \leqslant 1$, which holds trivially. Indeed, it is not difficult to see that in fact $\operatorname{cap}\left(S_{1 / 2,1 / 2}^{\tan }\right)=1$ since random bits are inserted at random positions in the sequence.

For $\delta_{0}=\delta_{1}=1$, this upper bound equals $H_{2}(1 / 3)=$ 0.9183 , which is the same as the upper bound given by

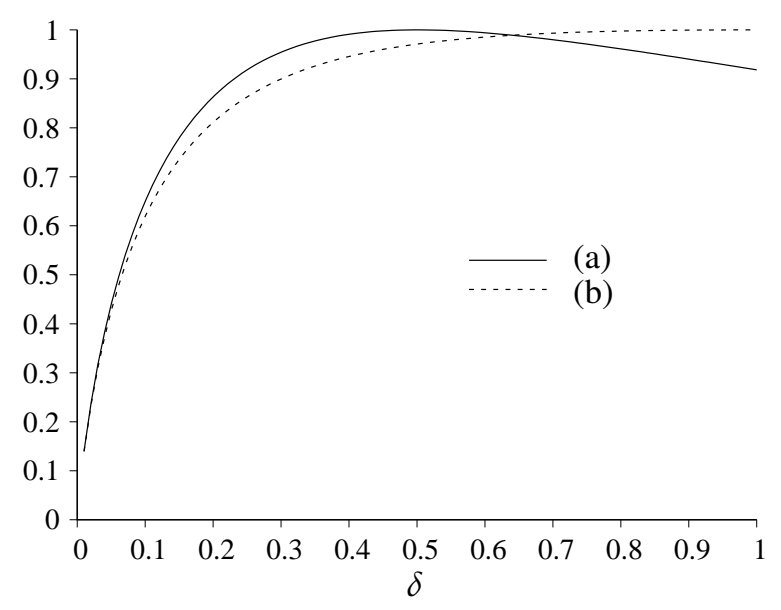

Figure 5. (a) An upper bound on $\operatorname{cap}\left(S_{\delta, \delta}^{\tan }\right)$, and (b) an upper bound on $\operatorname{cap}\left(S_{\delta}^{\text {tsb }}\right)$.

Theorem 11. The lower bound given by that theorem is 0.8689 , which indicates that for this case, the gap between the upper bound and the true value is small.

We also discuss a similar string-duplication system that has already been studied in [7], [15]. In general, such comparisons can be useful to decide between proposed mutation models for a given sequence, especially biological sequences. In that system, instead of tandem duplications that are probabilistically noisy, independent tandem duplications and substitutions are allowed. We compare the behavior of that system with $S_{\delta, \delta}^{\tan }$ for some $\delta \in[0,1]$. Specifically, we compare the bound of Theorem 13 for $\delta=\delta_{0}=\delta_{1}$,

$$
\operatorname{cap}\left(S_{\delta, \delta}^{\tan }\right) \leqslant H_{2}\left(\frac{1}{1+2 \delta}\right),
$$

with an upper bound for the system in which tandem duplications and substitutions occur with probabilities $1-\delta$ and $\delta$, respectively, at a random position in the sequence. We refer to this system as $S_{\delta}^{\text {tsb }}$. The definition of the capacity for $S_{\delta}^{\text {tsb }}$ is slightly different, to accommodate the fact that the length of the sequence does not necessarily grow in each step. It is shown in [15] that the capacity of this system is bounded from above by

$$
\operatorname{cap}\left(S_{\delta}^{\mathrm{tsb}}\right) \leqslant H_{2}\left(\frac{2 \delta}{1+3 \delta}\right) .
$$

The bounds are compared in Fig. 5 The bounds suggest that the systems behave differently when $\delta$ is away from 0 . In particular, $\operatorname{cap}\left(S_{\delta}^{\text {tsb }}\right) \leqslant 0.9709$ and $\operatorname{cap}\left(S_{\delta, \delta}^{\tan }\right)=1$ for $p=1 / 2$. For this value of $p$, in $S_{\delta}^{\text {tsb }}$ half of the mutations are duplications, which make substrings 00 and 11 more likely than what is expected in a random sequence, leading to capacity less than 1 .

\section{INTERSPERSED DUPLICATION}

Finally, we consider the case of interspersed duplication. While seemingly a more elaborate duplication rule (probabilistic both when choosing the bit to duplicate, as well as the 
insertion position), we now show that it has the same capacity as end duplication.

Theorem 14. Let $\Sigma=\{0,1\}, s \in \Sigma^{+}$be a seed string, and denote

$$
\begin{aligned}
S^{\text {end }} & =S_{\delta_{0}, \delta_{1}}^{\text {end }}=\left(\Sigma, s, T_{\delta_{0}, \delta_{1}}^{\text {end }}\right), \\
S^{\text {int }} & =S_{\delta_{0}, \delta_{1}}^{\text {int }}=\left(\Sigma, s, T_{\delta_{0}, \delta_{1}}^{\text {int }}\right) .
\end{aligned}
$$

Then

$$
\operatorname{cap}\left(S_{\delta_{0}, \delta_{1}}^{\mathrm{int}}\right)=\operatorname{cap}\left(S_{\delta_{0}, \delta_{1}}^{\mathrm{end}}\right)
$$

Proof: We first require some general arguments, in preparation for the proof for the capacity. Consider an interspersedduplication process, starting with the seed $s$, and running for $n$ mutation steps. Denote the bit generated in the $i$ th mutation step, $1 \leqslant i \leqslant n$, by $b_{|s|+i}$. We also use $b_{1} b_{2} \cdots b_{|s|}=s$ to denote the bits of the seed string.

The bits, however, do not appear in the order of generation (as they do in end duplication), since they are inserted in random places. Thus, if after $n$ mutations we reach a string $w \in \Sigma^{|s|+n}$, then

$$
w=b(\pi) \triangleq b_{\pi(1)} b_{\pi(2)} \cdots b_{\pi(|s|+n)},
$$

for some permutation $\pi \in \mathbb{S}_{|\mathcal{S}|+n}$ that satisfies

$$
\pi^{-1}(1)<\pi^{-1}(2)<\cdots<\pi^{-1}(|s|),
$$

since the order of the bits of the seed string is maintained. For example, we may have

$$
\begin{aligned}
s=S(0) & =b_{1} b_{2} b_{3} b_{4}=0011, \\
S(1) & =b_{1} b_{2} b_{3} b_{5} b_{4}=00101, \\
S(2) & =b_{1} \boldsymbol{b}_{6} b_{2} b_{3} b_{5} b_{4}=010101,
\end{aligned}
$$

and $\pi=[1,6,2,3,5,4]$.

Let us denote the set of permutations satisfying (12) by $P_{n}$, and hence, $\left|P_{n}\right|=(n+|s|) ! /|s|$ !. Since the insertion position at each mutation step is chosen independently and uniformly, the probability of each permutation is exactly,

$$
\frac{1}{|s|+1} \cdot \frac{1}{|s|+2} \cdots \frac{1}{|s|+n}=\frac{|s| !}{(n+|s|) !},
$$

i.e., the overall permutation is chosen uniformly from $P_{n}$.

Let us now denote,

$$
\begin{array}{ll}
t_{0} \triangleq|s|_{0}, & t_{1} \triangleq|s|-t_{0}, \\
k_{0} \triangleq\left|b_{|s|+1} \cdots b_{|s|+n}\right|_{0}, & k_{1} \triangleq n-k_{0},
\end{array}
$$

namely, $t_{0}$ and $t_{1}$ denote the number of zeros and ones (respectively) in the seed string, and $k_{0}$ and $k_{1}$ denote the number of zeros and ones (respectively) in the bits generated due to mutations.

We say $\pi_{1}, \pi_{2} \in P_{n}$ are equivalent, denoted $\pi_{1} \sim \pi_{2}$, if $b\left(\pi_{1}\right)=b\left(\pi_{2}\right)$. This is clearly an equivalence relation. For $\pi \in P_{n}$, let $E_{\pi}$ denote the equivalence class of $\pi$. Computing $\left|E_{\pi}\right|$ is hard, but it suffices for us to bound it by

$$
k_{0} ! k_{1} ! \leqslant\left|E_{\pi}\right| \leqslant\left(t_{0}+k_{0}\right) !\left(t_{1}+k_{1}\right) !
$$

For the lower bound, we permute only the newly generated zeros between themselves, and similarly the ones, while keeping the bits of the seed in their place. For the upper bound, we permute all zeroes between themselves, and similarly the ones, thus, perhaps reaching some permutations that are not in $P_{n}$.

Lastly, denote by $A_{k_{0}}$ the event that that among the $n$ bits generated due to mutations, exactly $k_{0}$ are zeros, and the rest, $k_{1}=n-k_{0}$ are ones. Also, let $B^{\text {int }}\left(n, k_{0}\right)$ denote the set of strings $w \in \Sigma^{|s|+n},|w|_{0}=k_{0}+t_{0}$, that may be obtained from $s$ using $n$ interspersed-duplication mutations. It then follows that if $w \in B^{\text {int }}(n)$, then

$$
\begin{gathered}
\frac{\left(t_{0}+t_{1}\right) ! k_{0} ! k_{1} !}{\left(t_{0}+t_{1}+k_{0}+k_{1}\right) !} \operatorname{Pr}\left(A_{k_{0}}\right) \leqslant \operatorname{Pr}\left(S^{\mathrm{int}}(n)=w\right) \\
\leqslant \frac{\left(t_{0}+t_{1}\right) !\left(t_{0}+k_{0}\right) !\left(t_{1}+k_{1}\right) !}{\left(t_{0}+t_{1}+k_{0}+k_{1}\right) !} \operatorname{Pr}\left(A_{k_{0}}\right) .
\end{gathered}
$$

This means that

$$
\operatorname{Pr}\left(S^{\text {int }}(n)=w\right)=\operatorname{Pr}\left(A_{k_{0}}\right) \cdot 2^{-n\left(H_{2}\left(k_{0} / n\right)+o(1)\right)},
$$

as well as

$$
\left|B^{\text {int }}\left(n, k_{0}\right)\right|=2^{n\left(H_{2}\left(k_{0} / n\right)+o(1)\right)} .
$$

We are now ready to prove our claims. First, we look at the noiseless case, $\delta_{0}=\delta_{1}=0$. The probability, $\operatorname{Pr}\left(A_{k_{0}}\right)$ has already been given in (2). We therefore get,

$$
\begin{aligned}
\operatorname{cap}\left(S^{\text {int }}\right) & =\limsup _{n \rightarrow \infty} \frac{1}{n} H\left(S^{\text {int }}(n)\right) \\
& =\limsup _{n \rightarrow \infty} \frac{1}{n} \sum_{k_{0}=0}^{n} \operatorname{Pr}\left(A_{k_{0}}\right) \log _{2}\left|B^{\text {int }}\left(n, k_{0}\right)\right| \\
& =\int_{0}^{1} \beta\left(p ; t_{0}, t_{1}\right) H_{2}(p) \mathrm{d} p \\
& =\operatorname{cap}\left(S^{\text {end }}\right)
\end{aligned}
$$

exactly as in the proof of Theorem 3 .

The second (and last) case is $\delta_{0}+\delta_{1}>0$. Denote $\alpha \triangleq$ $\delta_{1} /\left(\delta_{0}+\delta_{1}\right)$. By Lemma 7, for any $\epsilon_{1}, \epsilon_{2}>0$, there exists $N \in \mathbb{N}$ such that for all $n \geqslant N, \operatorname{Pr}\left(\left|\operatorname{fr}_{0}\left(S^{\operatorname{int}}(n)\right)-\alpha\right| \leqslant\right.$ $\left.\epsilon_{1}\right) \geqslant 1-\epsilon_{2}$. Then,

$$
\begin{aligned}
& \operatorname{cap}\left(S^{\text {int }}\right) \\
& =\limsup _{n \rightarrow \infty} \frac{1}{n} H\left(S^{\text {int }}(n)\right) \\
& =\limsup _{n \rightarrow \infty} \frac{1}{n}\left(\sum_{\left|\frac{k_{0}+t_{0}}{n+|s|}-\alpha\right| \leqslant \epsilon_{1}} \operatorname{Pr}\left(A_{k_{0}}\right) \log _{2}\left|B^{\text {int }}\left(n, k_{0}\right)\right|\right. \\
& \left.\quad+\sum_{\left|\frac{k_{0}+t_{0}}{n+|s|}-\alpha\right|>\epsilon_{1}} \operatorname{Pr}\left(A_{k_{0}}\right) \log _{2}\left|B^{\text {int }}\left(n, k_{0}\right)\right|\right) \\
& \leqslant \max _{x \in\left[\alpha-\epsilon_{1}, \alpha+\epsilon_{1}\right]} H_{2}(x)+\epsilon_{2} .
\end{aligned}
$$


On the other hand,

$$
\begin{aligned}
& \operatorname{cap}\left(S^{\text {int }}\right) \\
& =\limsup _{n \rightarrow \infty} \frac{1}{n} H\left(S^{\text {int }}(n)\right) \\
& =\limsup _{n \rightarrow \infty} \frac{1}{n}\left(\sum_{\left|\frac{k_{0}+t_{0}}{n+|s|}-\alpha\right| \leqslant \epsilon_{1}} \operatorname{Pr}\left(A_{k_{0}}\right) \log _{2}\left|B^{\text {int }}\left(n, k_{0}\right)\right|\right. \\
& \left.\quad+\sum_{\left|\frac{k_{0}+t_{0}}{n+|s|}-\alpha\right|>\epsilon_{1}} \operatorname{Pr}\left(A_{k_{0}}\right) \log _{2}\left|B^{\text {int }}\left(n, k_{0}\right)\right|\right) \\
& \geqslant\left(1-\epsilon_{2}\right) \min _{x \in\left[\alpha-\epsilon_{1}, \alpha+\epsilon_{1}\right]} H_{2}(x) .
\end{aligned}
$$

Taking the limit of (13) and (14) as $\epsilon_{1}, \epsilon_{2} \rightarrow 0^{+}$, we obtain

$$
\operatorname{cap}\left(S^{\text {int }}\right)=H_{2}(\alpha)=\operatorname{cap}\left(S^{\text {end }}\right),
$$

as claimed.

\section{CONCLUSION}

In this paper we defined and studied three Pólya string models. We determined the exact capacity of end duplication, $S_{\delta_{0}, \delta_{1}}^{\text {end }}$, and interspersed duplication, $S_{\delta_{0}, \delta_{1}}^{\text {int }}$, both for any noise parameters $\delta_{0}$ and $\delta_{1}$. We also found the exact capacity of noiseless tandem duplication, $S_{0,0}^{\tan }$, as well as we connected the capacity of complement tandem duplication, $S_{1,1}^{\text {tan }}$, with the signatures of random permutations. Finally, we upper bounded the capacity of general noisy tandem duplication, $S_{\delta_{0}, \delta_{1}}^{\tan }$.

We make several interesting observation. First, had we used a Pólya urn model instead of a string model, then no difference would have been observed between tandem and end duplication. Indeed, the distribution of 0's and 1's in both cases is the same. However, when considering the structure of a string, the difference between the two comes to light.

Many other differences are apparent between the combinatorial capacity (found in [6]) and the probabilistic capacity studied here, and we point a few:

- While the combinatorial capacity of (noiseless) end duplication is known to be 1 , in the probabilistic model it varies depending on the starting string.

- Similarly, for the complement tandem-duplication model, it is easy to show that the combinatorial capacity is 1 , while the probabilistic capacity is bounded away from both 0 and 1 .

- The probabilistic capacity of $S_{\delta_{0}, \delta_{1}}^{\text {end }}$ is equal to that of $S_{\delta_{0}, \delta_{1}}^{\text {int }}$, which is not generally the case when using the combinatorial capacity.

Many open questions remain. Obvious ones include the determination of $\operatorname{cap}\left(S_{\delta_{0}, \delta_{1}}^{\mathrm{tan}}\right)$ for all values of $\delta_{0}$ and $\delta_{1}$. We also note that the systems studied in the current paper are limited to duplications of length 1 , while genomic duplication mutations are observed for a large range of duplication lengths. Thus, extending the results to longer duplication lengths is an important open task. Other noise models are also of interest. For example, one might be interested in models in which mutation steps either duplicate or substitute a letter (e.g., see [7], [15]). Finally, more elaborate distributions may be studied, including context-sensitive duplication rules.

\section{REFERENCES}

[1] N. Alon, J. Bruck, F. Farnoud, and S. Jain, "Duplication distance to the root for binary sequences," IEEE Trans. Inform. Theory, vol. 63, no. 12, pp. 7793-7803, Dec. 2017.

[2] S. Banach, "Sur les opérations dans les ensembles abstraits et leur application aux équations intégrales," Fund. Math, vol. 3, no. 1, pp. 133-181, 1922.

[3] V. Borkar, Stochastic Approximation - A Dynamical System Viewpoint. Cambridge University Press, 2008.

[4] Y. M. Chee, J. Chrisnata, H. M. Kiah, and T. T. Nguyen, "Deciding the confusability of words under tandem repeats," arXiv preprint arXiv:1707.03956, 2017

[5] F. Farnoud, M. Schwartz, and J. Bruck, "A stochastic model for genomic interspersed duplication," in Proceedings of the 2015 IEEE International Symposium on Information Theory (ISIT2015), Hong Kong, China SAR, Jun. 2015, pp. 1731-1735.

[6] —-, "The capacity of string-duplication systems," IEEE Trans. Inform. Theory, vol. 62, no. 2, pp. 811-824, Feb. 2016.

[7] — "Estimation of the duplication history under a stochastic model for tandem repeats," 2018, in preparation.

[8] J. W. Fondon and H. R. Garner, "Molecular origins of rapid and continuous morphological evolution," Proceedings of the National Academy of Sciences, vol. 101, no. 52, pp. 18 058-18 063, 2004.

[9] S. Jain, F. Farnoud, and J. Bruck, "Capacity and expressiveness of genomic tandem duplication," IEEE Trans. Inform. Theory, vol. 63, no. 10, pp. 6129-6138, Oct. 2017.

[10] S. Jain, F. Farnoud, M. Schwartz, and J. Bruck, "Duplication-correcting codes for data storage in the DNA of living organisms," IEEE Trans. Inform. Theory, vol. 63, no. 8, pp. 4996-5010, Aug. 2017.

[11] A. Jeffrey and H.-H. Dai, Handbook of Mathematical Formulas and Integrals, 4th Edition. Elsevier, 2008.

[12] E. S. Lander, L. M. Linton, B. Birren, C. Nusbaum, M. C. Zody, J. Baldwin, K. Devon, K. Dewar, M. Doyle, W. FitzHugh et al., "Initial sequencing and analysis of the human genome," Nature, vol. 409, no. 6822, pp. 860-921, 2001.

[13] A. Lenz, N. Jünger, and A. Wachter-Zeh, "Bounds and constructions for multi-symbol duplication error correcting codes," arXiv preprint arXiv:1807.02874, 2018.

[14] P. Leupold, V. Mitrana, and J. M. Sempere, "Formal languages arising from gene repeated duplication," in Aspects of Molecular Computing. Springer, 2004, pp. 297-308.

[15] H. Lou, M. Schwartz, and F. Farnoud, "Evolution of N-gram frequencies under duplication and substitution mutations," in Proceedings of the 2018 IEEE International Symposium on Information Theory (ISIT2018), Vail, CO, USA, Jun. 2018, pp. 2246-2250.

[16] F. J. MacWilliams and N. J. A. Sloane, The Theory of Error-Correcting Codes. North-Holland, 1978.

[17] H. Mahmoud, Pólya Urn Models, 1st ed. Chapman \& Hall/CRC, 2008.

[18] N. I. Mundy and A. J. Helbig, "Origin and evolution of tandem repeats in the mitochondrial DNA control region of shrikes (lanius spp.)," Journal of Molecular Evolution, vol. 59, no. 2, pp. 250-257, 2004.

[19] S. L. Shipman, J. Nivala, J. D. Macklis, and G. M. Church, "CRISPRCas encoding of digital movie into the genomes of a population of living bacteria," Nature, vol. 547, pp. 345-349, Jul. 2017.

[20] K. Usdin, "The biological effects of simple tandem repeats: lessons from the repeat expansion diseases," Genome Research, vol. 18, no. 7, pp. 1011-1019, 2008.

[21] Y. Yehezkeally and M. Schwartz, "Reconstruction codes for DNA sequences with uniform tandem-duplication errors," in Proceedings of the 2018 IEEE International Symposium on Information Theory (ISIT2018), Vail, CO, USA, Jun. 2018, pp. 2535-2539. 